\title{
Soccer Event Recognition Technique based on Pattern Matching
}

\author{
Jiwon Lee, Do-won Nam, Sungwon Moon, JungSoo Lee, and Wonyoung Yoo \\ SW.Content Research Laboratory, \\ Electronics and Telecommunications Research Institute (ETRI), \\ 218 Gajeong-ro, Yuseong-gu, Daejeon, Republic of Korea \\ Email: \{ez1005, dwnam, moonstarry, jslee2365, zero2\}@etri.re.kr
}

\begin{abstract}
Recently, there has been an increasing number of attempts to analyze sport activities through the combination of sports science and ICT technology. In the case of soccer, several leading companies have already developed tracking techniques for players to automatically acquire sports analysis data. However, the automatic extraction of event data for analyzing the sports games is limited to the level of academic research, and the field still depends on the manual work of professional analysts. This paper proposes a soccer event recognition technology based on pattern matching. As can be seen from the experimental results, it is possible to recognize various events much more accurately than the event recognition technology at academic research level.
\end{abstract}

\section{INTRODUCTION}

$\mathbf{R}$ ECENTLY, a case attempting to analyze sports activity through sports science and ICT technology combines increased. In particular, in the case of soccer and baseball games, as shown in Table I, several leading international companies have already developed tracking techniques for players and balls to automatically acquire sports analysis data [1]. However, the automatic extraction of event data for analyzing the contents of sports games is a mere academic research level. Especially, in the case of soccer games, it is still dependent on the manual work of professional recorders and analysts.

TABLE I

Major COMMercial Sports ANALYSis System

\begin{tabular}{|c|c|l|}
\hline Products & Sports & \multicolumn{1}{c|}{ Special features } \\
\hline \hline TRACAB & Soccer & $\begin{array}{l}\text { Video-based real-time 3D player tracking tech- } \\
\text { nique created using rocket tracking technology }\end{array}$ \\
\hline Viper & Soccer & $\begin{array}{l}\text { Sensor-based technology to measure player's } \\
\text { speed, acceleration, maximum speed and heart } \\
\text { rate during sports game }\end{array}$ \\
\hline Prozone & Soccer & $\begin{array}{l}\text { Player tracking and real-time game analysis } \\
\text { based on big data }\end{array}$ \\
\hline $\begin{array}{c}\text { Club } \\
\text { Portal }\end{array}$ & Soccer & $\begin{array}{l}\text { Real-time player tracking and handwriting input } \\
\text { based event extraction }\end{array}$ \\
\hline Hawk-Eye & $\begin{array}{l}\text { Soccer } \\
\text { Tennis }\end{array}$ & $\begin{array}{l}\text { Goal line out detection based on high-speed } \\
\text { multi cameras }\end{array}$ \\
\hline SportVision & Baseball & $\begin{array}{l}\text { Player/Ball recognition and tracking in MLB by } \\
\text { using the composition of radar and video-based } \\
\text { tracking technology }\end{array}$ \\
\hline FreeD & Baseball & $\begin{array}{l}\text { 3D play motion reconstruction by using high- } \\
\text { speed multi cameras }\end{array}$ \\
\hline
\end{tabular}

In this paper, we propose a soccer event recognition technique based on pattern matching that can solve the problems of existing academic research and apply it to real field of soccer event recognition technique.

The composition of this paper is as follows. In Sec. II, we describe the existing researches. In Sec. III, we propose automatic pattern matching based soccer event recognition. Sec. IV shows the experimental results of the proposed method. Finally, Sec. V discusses the conclusions and future research directions.

\section{Previous Works}

The existing event recognition techniques using soccer video are largely based on video-shot and voice based. First, in the case of video-shot based event recognition, the sequence of the method of shooting the game for each event in the soccer broadcast video is defined in advance for recognizing the goal, shooting, corner kick, free kick, penalty kick and foul [2], [3]. That is, firstly, the given soccer broadcast video is classified into a close-up view, a short view, a long view, and a crowd view, and then a pattern of each view is displayed for each event. On the other hand, in the case of voice-based event recognition, a method of recognizing the occurrence of important events by recognizing the referee whistle sound and the voice loudness and style of the commentator and spectators was used [4].

The above conventional methods are not based on the action of the object such as the player/referee who perform the actual game, but rather recognize audio/video clues in broadcast soccer video and recognize main event based on it. Therefore, it has a limitation that it can not sufficiently reflect the change of the shooting technique or the reaction pattern of the spectator and the commentator which is different from the predefined pattern. In addition, the existing technique can not recognize the event itself because it does not accurately understand the movement and motion of the player/referee. Therefore, rather than classifying and analyzing each event accurately, it has a limitation that it focuses more on producing highlight video by grouping the recognized points on the assumption that the main event will exist at the recognized point in time.

Taking all the above into consideration, we need a way to accurately identify soccer events through the position and 


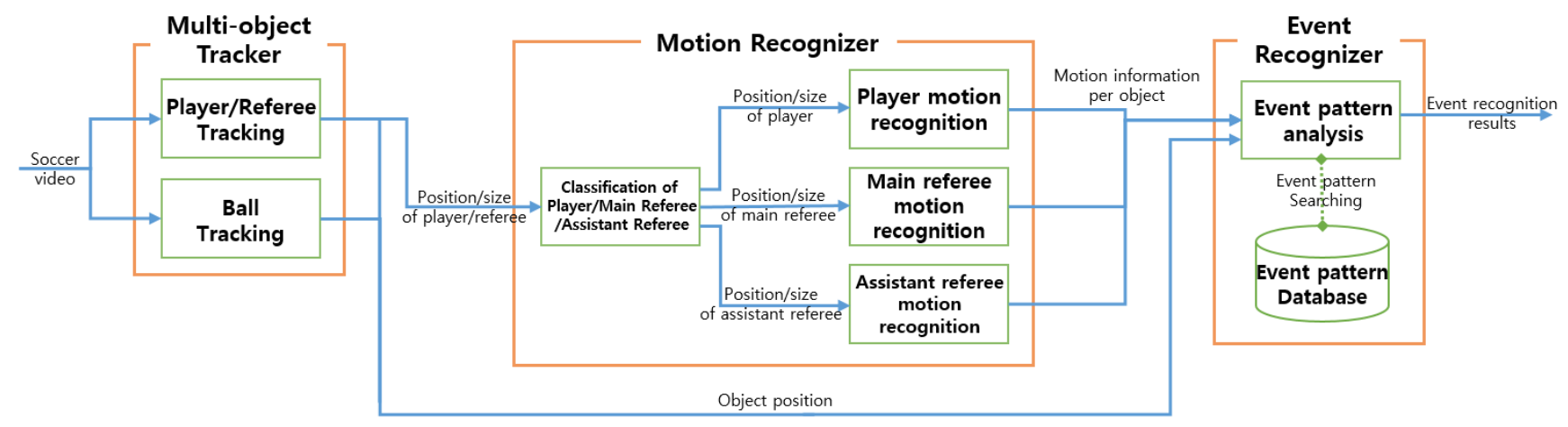

Fig. 1. Block diagram for soccer event recognition system

motion information of the objects directly related to the game, such as the players/referees/ball, not the indirect clues related to the game, such as the camera movement and the response of the spectators/commentators.

\section{Proposed Method}

The proposed event recognition system is shown in Fig. 1. As can be seen in the figure, the proposed soccer event recognition system comprises multi-object tracking unit for recognizing the position and size of the players/referees/ball from the given video, a motion recognition unit for classifying each tracked object, and an event recognition unit for recognizing the event on the basis of the recognized per-object motion and position information. That is, in order to recognize the event in the soccer game by using the proposed method, techniques development for multi-object tracking and motion recognition should be preceded.

The multi-object tracking unit receives the soccer video as input and extracts the position and size information of the players/referees/ball in the game and delivers it to the next stage. Here, the tracker for the players/referees and the tracker for the balls are classified into two modules because they should be developed on the basis of different algorithms based on the characteristics of the objects.

The motion recognition unit receives the tracking information of the players/referees as an input, first classifies

TABLE II

MOTION LIST FOR EACH OBJECT CLASSIFIER

\begin{tabular}{|c|c|c|}
\hline Main referee & Assistant referee & Field player \\
\hline $\begin{array}{c}\text { Walking without } \\
\text { hand gestures }\end{array}$ & $\begin{array}{c}\text { Walking without } \\
\text { flag gestures }\end{array}$ & Walking \\
\hline $\begin{array}{c}\text { Running without } \\
\text { hand gestures }\end{array}$ & $\begin{array}{c}\text { Running without } \\
\text { flag gestures }\end{array}$ & Running \\
\hline $\begin{array}{c}\text { Pointing with } \\
\text { one arm }\end{array}$ & $\begin{array}{c}\text { Walking sideways } \\
\text { without flag gestures }\end{array}$ & $\begin{array}{c}\text { Corner Kick } \\
\text { Free Kick }\end{array}$ \\
\hline Lifting yellow card & $\begin{array}{c}\text { Lifting the flag } \\
\text { over the head }\end{array}$ & $\begin{array}{c}\text { Kick with } \\
\text { wielding arm }\end{array}$ \\
\hline Lifting red card & $\begin{array}{c}\text { Lifting the flag } \\
\text { to the chest height } \\
\text { Pointing } \\
\text { with the flag }\end{array}$ & Throw in \\
\hline & & Lying down \\
\hline
\end{tabular}

TABLE III

EXAMPLES OF EVENT RECOGNITION PATTERN

\begin{tabular}{|c|c|}
\hline Event & Examples of recognition pattern \\
\hline Shooting & $\begin{array}{l}\text { The player had a ball } \\
\rightarrow \text { The player made a kick motion } \\
\rightarrow \text { The ball moved in the direction of the goalpost }\end{array}$ \\
\hline Pass & $\begin{array}{l}\text { The player had a ball } \\
\rightarrow \text { The player made a kick motion } \\
\rightarrow \text { The ball moved to the same team player }\end{array}$ \\
\hline Tackle & $\begin{array}{l}\text { The player made a walking or running motion } \\
\rightarrow \text { The player made a lying down motion } \\
\rightarrow \text { The opponent with a similar position }\end{array}$ \\
\hline Corner kick & $\begin{array}{l}\text { The positions of player and ball are similar and near the } \\
\text { corner kick position } \\
\rightarrow \text { The player made a kick motion } \\
\rightarrow \text { The ball moved (to anywhere) }\end{array}$ \\
\hline Free kick & $\begin{array}{l}\text { The main referee made a pointing with one arm motion } \\
\rightarrow \text { The positions of player and ball are similar } \\
\rightarrow \text { The player made a kick motion } \\
\rightarrow \text { The ball moved (to anywhere) }\end{array}$ \\
\hline Penalty kick & $\begin{array}{l}\text { The main referee made a pointing with one arm motion } \\
\rightarrow \text { The positions of player and ball are similar and near } \\
\text { the penalty kick spot } \\
\rightarrow \text { The player made a kick motion } \\
\rightarrow \text { The ball moved in the direction of the goalpost }\end{array}$ \\
\hline Offside & $\begin{array}{l}\text { The assistant referee made a lifting the flag over the head } \\
\text { motion } \\
\rightarrow \text { The assistant referee made a lifting the flag to the chest } \\
\text { height motion }\end{array}$ \\
\hline Foul & The main referee made a pointing with one arm motion \\
\hline Card & The main referee made a lifting yellow or red card motion \\
\hline Assist & $\begin{array}{l}\text { Recognizing the pass event } \\
\rightarrow \text { Recognizing the shooting event } \\
\rightarrow \text { The position of ball is inside the goal line }\end{array}$ \\
\hline $\begin{array}{c}\text { Player } \\
\text { substitution }\end{array}$ & $\begin{array}{l}\text { The main referee made a pointing with one arm motion } \\
\rightarrow \text { One player leaves the field } \\
\rightarrow \text { Another player from the same team enters the field }\end{array}$ \\
\hline
\end{tabular}

each object by the players/main referee/assistant referees, and performs motion recognition for each classification. Table II shows the motion list for each object classifier that will be recognized by the motion recognition unit in order to finally aim at event recognition.

The event recognizing unit finds the sequence of motion and position information of objects which is similar to a predefined event pattern inside the event pattern database through a pattern matching technique and outputs it as a recognized 

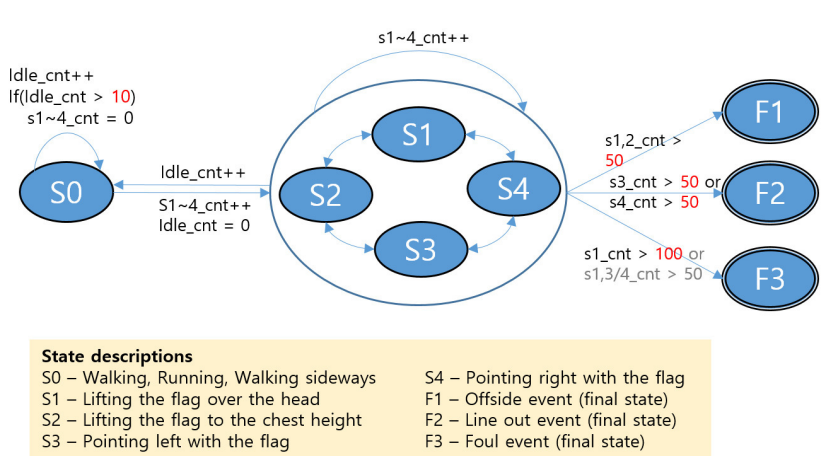

Fig. 2. Derived assistant referee DFA for soccer event recognition

soccer event. Here, the events to be recognized in this paper are the 11 major soccer events being defined and used by the Korea Professional Soccer Federation. Table III shows some examples of patterns for each event included in the event pattern database [5].

If we look at the characteristics of the pattern defined in this table, it can be seen that an arbitrary state can be expressed by integrating the position and motion information of the object. Then, if the state moves from one state to the next state and reaches the final state, it can be seen that the pattern is defined so that the event can be recognized. Through this consideration, we derived a deterministic finite automata (DFA) consisting of states and transitions from event patterns to implement a pattern-based event recognizer. An example of the derived event DFA is shown in Fig. 2.

\section{EXPERIMENTAL RESULTS}

In order to develop the proposed soccer event recognition technique and to test its performance, it is necessary to develop a multi-object tracker and a motion recognizer. For this purpose, we implemented the multi-object tracking technique proposed by Kim et al. [6] and the deep learning based soccer object motion recognizer proposed by Lee et al. [7]

As videos for this experiment, we selected 3 games of the K League Challenge 2016 and conducted the experiment. The game was taken at $4 \mathrm{~K} 30 \mathrm{fps}$, and the camera was installed in a fixed form and the entire area of the stadium was recorded with one camera. Snapshot samples of the captured video are shown in Fig. 3.

In the DFA configuration for event recognition, ownership of the ball was the most important issue. In the paper, the simplest assumption is that the corresponding object has a ball whose distance between the ball and the object is below the threshold. If there are more than two objects below the threshold, it is assumed that the closer object has the ball. The threshold setting for the experiment was set to 0.5 meters.

The finally derived DFAs are three, and the key object of each DFA is main referee, assistant referee, and ball respectively. The main referee DFA was designed to recognize foul and card events, and the assistant referee DFA was designed to recognize offside and foul events. In the case
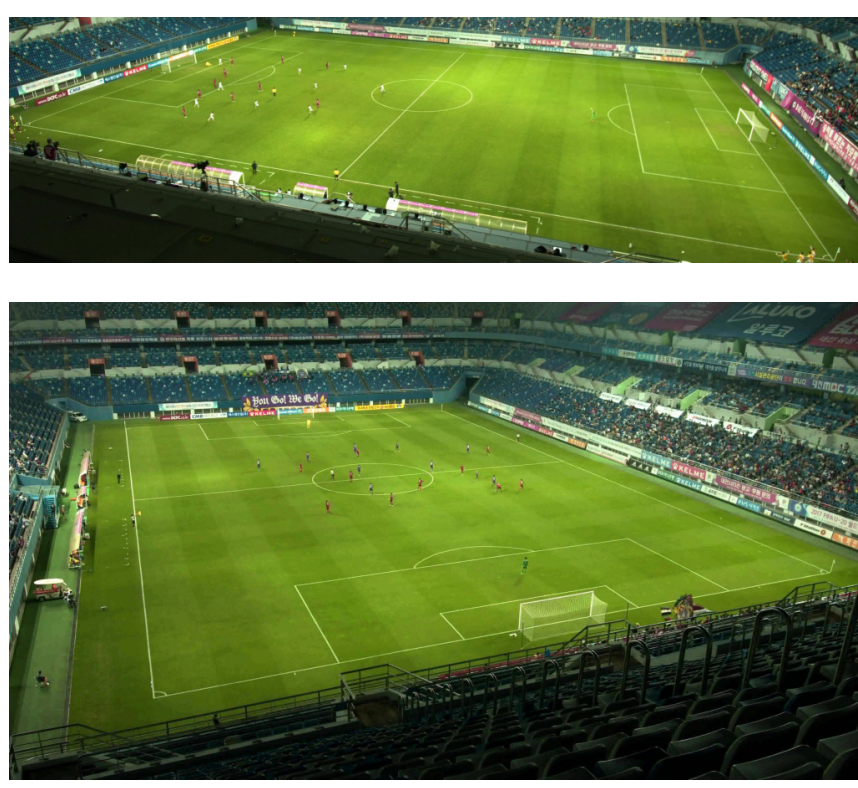

Fig. 3. Snapshot samples of the K League challenge 2016 for the experiments

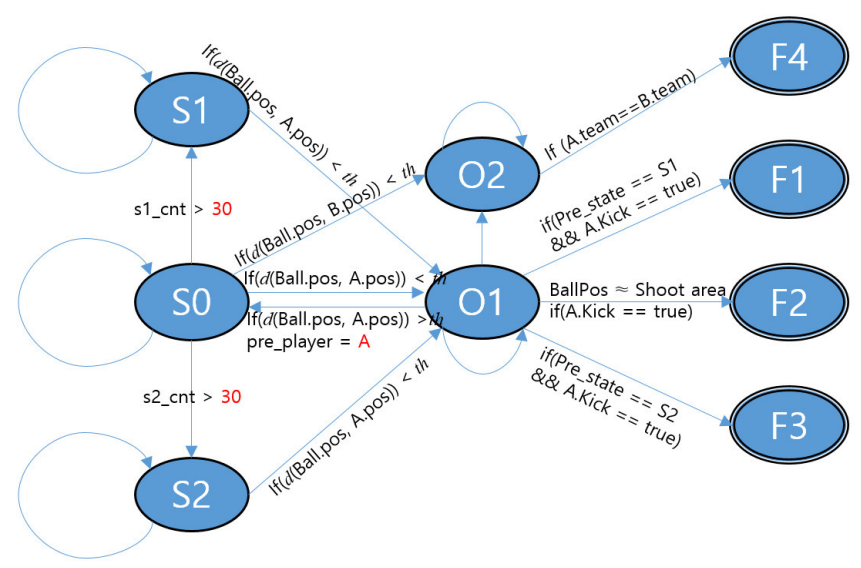

State descriptions

SO - No one own the ball

S1 - No one own the ball \& The ball is around the penalty position

$\mathrm{S} 2$ - No one own the ball \& The ball is around the corner kick position

01 - Player A owns the ball

O2 - Player B owns the ball

F1 - Penalty kick event (final state)

F2 - Shooting event (final state)

F3 - Corner kick event (final state)

F4 - Pass event (final state)

Fig. 4. Derived ball DFA for soccer event recognition

of ball DFA, it was designed to recognize shooting, pass, corner kick, penalty kick, and assist events. In the case of tackle and player substitution events, it is difficult to define the relationship among multiple objects. Thus, we excluded those events recognition attempt in this paper. The derived assistant referee DFA is shown in Fig. 2, and the ball DFA is shown in Fig. 4. In the case of the referee DFA, the complexity is very simple so it is not included in this paper. 
TABLE IV

RESULTS OF PROPOSED SOCCER EVENT RECOGNIZER

\begin{tabular}{|c|c|c|c|}
\hline Events & $\begin{array}{c}\text { The number } \\
\text { of occurrences }\end{array}$ & $\begin{array}{c}\text { The number } \\
\text { of recognitions }\end{array}$ & $\begin{array}{c}\text { Recognition } \\
\text { rate }\end{array}$ \\
\hline \hline Shooting & 14 & 7 & $50 \%$ \\
\hline Pass & 35 & 21 & $60 \%$ \\
\hline Corner kick & 18 & 11 & $61.1 \%$ \\
\hline Free kick & 10 & 4 & $40 \%$ \\
\hline Penalty kick & 3 & 3 & $100 \%$ \\
\hline Offside & 15 & 14 & $93.3 \%$ \\
\hline Foul & 17 & 10 & $58.8 \%$ \\
\hline Card & 5 & 4 & $80 \%$ \\
\hline Assist & 3 & 1 & $33.3 \%$ \\
\hline
\end{tabular}

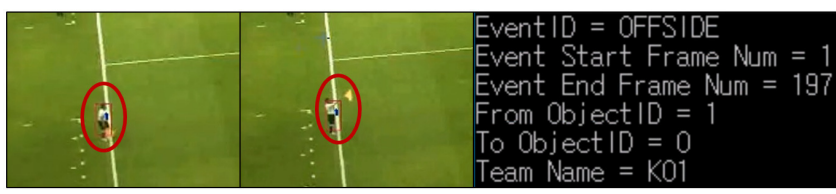

(a) Offside event recognition by assistant referee DFA (before, after, result)

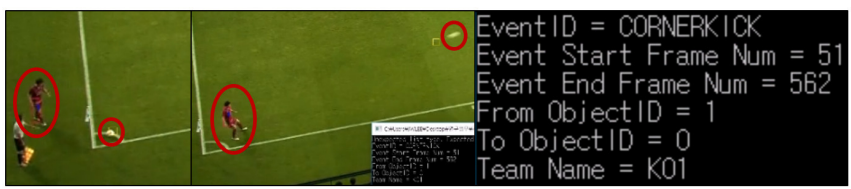

(b) Corner kick event recognition by ball DFA (before, after, result)

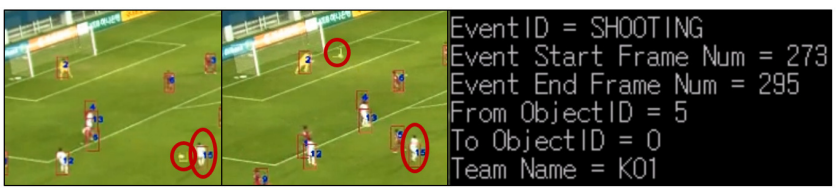

(c) Shooting event recognition by ball DFA (before, after, result)

Fig. 5. Example of soccer event recognition result based on pattern matching

Table IV and Fig. 5 show the results of the soccer event recognition experiment through the assistant referee and Ball DFAs. The table shows that the simpler the object motion and the fewer the related objects, the higher the accuracy of event recognition. Also, as shown in the figure, the proposed event recognizer accurately reports detailed information such as the start/end points of the event and the related object position, unlike the level of existing academic researches, when the event recognition is successful.

However, the proposed technique does not have enough event recognition accuracy. This is mainly due to the performance problems of multi-object tracker and motion recognizer, and the lack of available event patterns. In order to recognize the accurate event, the position of the objects obtained through the multi-object tracker must be precise, and then the motions of the recognized object must be accurately recognized through the motion recognizer. However, the tracking accuracy of the current tracker is about $90 \%$ for the player/referee and about $70 \%$ for the ball, and the recognition accuracy of the motion recognizer is about $85 \%$ for the recognized tracking result. Therefore, the cumulative errors in these two parts will inevitably affect the accuracy of event recognizer. In addition, there are cases in which the recognition pattern of each soccer event has not been enough so that the event occurrence situation is not recognized. These problems are expected to gradually improve over time.

\section{Conclusion and Future Directions}

Along with the trend to integrate ICT technology into the sports area, it is also attempting to understand the game clearly by extracting low level statistics in the soccer game. Of course, attempts to automate high-level event recognition through ICT technology are ongoing, but it is still in the hands of skilled professionals.

In this paper, we propose a method to recognize specific event type, occurrence timing, and generated object by using event pattern matching technique based on multi-object positions and motion information from soccer game video. Therefore, it is possible to recognize more specific events than conventional techniques that recognize only main events using shooting pattern or audio information for event recognition.

In the future, we will further refine the proposed technique and try to recognize a higher level of soccer game strategy that can be composed of a set of position/motion/event. In addition, if the original idea of the proposed technology is appropriately used, it will be very useful not only in the soccer game, but also in the video understanding application such as the analysis of other sport fields and CCTV based human behavior analysis in which similar patterns of events occur.

\section{ACKNOWLEDGMENT}

This research is supported by Ministry of Culture, Sports and Tourism(MCST) and Korea Creative Content Agency(KOCCA) in the Culture Technology(CT) Reasearch \& Development Program 2016 (R2016030044, Development of Context-Based Sport Video Analysis, Summarization, and Retrieval Technologies)

\section{REFERENCES}

[1] J. Lee, D. W. Nam, J. S. Lee, S. Moon, K. Kim, and H. Kim, "A Study on Composition of Context-based Soccer Analysis System," in Proc. ICACT 2017, pp. 886-889, Feb. 2017.

[2] T. Y. Lee, "A Detecting Method and a Training Method of Event for Soccer Video," in Republic of Korea patent, patent registration number 10-0963744, May. 2010.

[3] A. Ekin, A. M. Tekalp, and R. Mehrotra, "Automatic soccer video analysis and summarization," IEEE trans. Image processing, vol. 12, pp. 796-807, http://dx.doi.org/10.1109/TIP.2003.812758, Jul. 2003.

[4] M. Xu, N. C. Maddage, C. Xu, and Q. Tian, "Creating audio keywords for event detection in soccer video," in Proc. ICME, pp. I-281-II-284, Jul. 2003.

[5] J. Lee, D. W. Nam, S. Moon, J. S. Lee, and H. Kim, "Apparatus and Method for Soccer Event Detection Based on Motion," in Republic of Korea patent, patent application number 10-2016-0167319, Dec. 2016.

[6] W. J. Kim, S. Moon, D. W. Nam, and H. Kim, "Method and Device for Tracking Multiple Objects," in Republic of Korea patent, patent application number 10-2016-0113911, Sep. 2016.

[7] J. Lee, S. Moon, D. W. Nam, and H. Kim, "Apparatus and Method for Recognizing Motion in Video," in Republic of Korea patent, patent application number 10-2016-0169344, Dec. 2016. 